\title{
Teachers' and Parents' Perspectives on the Feasibility of a Preschool-Based Behavioral Intervention to Prevent Obesity: An Embedded Qualitative Study within ToyBox Study Malaysia
}

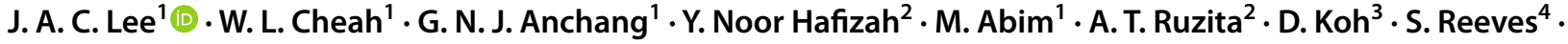 \\ C. Summerbell ${ }^{5,6} \cdot$ C. A. Essau ${ }^{3}$ B. K. Poh ${ }^{2}$.E. L. Gibson ${ }^{3}$
}

Accepted: 19 November 2021

(c) Springer Nature B.V. 2021

\begin{abstract}
The aim of this qualitative study, an embedded component of ToyBox Study Malaysia (TSM), was to elicit the perspectives of teachers and parents regarding the implementation of TSM. TSM is a preschool-based behavioral intervention program aimed at improving healthy energy balance-related behaviors among young children attending preschools. The qualitative study adopted a descriptive-interpretive methodology, and triangulated data collected through semi-structured focus groups with artifacts collected. The setting involved rural and metropolitan preschools in Sarawak and Peninsular Malaysia, respectively. In Sarawak, 11 teachers and 20 parents from six intervention preschools participated in this study. In Peninsular Malaysia, 14 preschool teachers and seven assistant teachers representing all 15 intervention preschools participated in the study. Data were analyzed thematically and four overarching themes were identified: impact of TSM on the children's knowledge and practices of healthy energy balance-related behaviors; spheres of influence upon the children's energy balance-related behaviors; constraints and affordances related to the implementation of TSM; and prospective sustainability of TSM. The triangulation of data from teachers, parents, and the artifacts related to TSM enabled the corroboration of evidence to support the themes identified. This study provides evidence on the reciprocal interactions between the teachers and parents who played key roles in facilitating behavioral change in the children, and the children who, in turn, served as change agents beyond the preschool. Furthermore, the mediational tools such as the TSM crockery and availability of healthy food and water led to the reported behavioral changes at both rural and metropolitan settings.
\end{abstract}

Keywords Preschool $\cdot$ Obesity $\cdot$ Healthy eating $\cdot$ Physical activity $\cdot$ Early care and education prevention $\cdot$ Public health

\section{Introduction}

Childhood obesity is a global problem of the twenty-first century (World Health Organization [WHO], 2016). Being overweight and obese in childhood poses serious public

\footnotetext{
J. A. C. Lee

aclee@unimas.my

1 Universiti Malaysia Sarawak, Kota Samarahan, Malaysia

2 Universiti Kebangsaan Malaysia, Kuala Lumpur, Malaysia

3 Universiti Kebangsaan Malaysia, Bangi, Malaysia

4 University of Roehampton, London, UK

5 Durham University, Durham, UK

6 Fuse, NIHR Centre for Translational Research in Public Health, Newcastle upon Tyne, UK
}

health consequences and health related risks such as high blood pressure, high cholesterol, and Type 2 diabetes, psychological problems such as depression and anxiety, and antisocial risks such as bullying, stigma, and social isolation (Beck, 2016; Centers for Disease Control \& Prevention, 2020).

Childhood obesity is also related to reduced academic performance (OECD, 2019). Healthy-weight children are more likely, compared with children who are obese, to attain greater educational grades in school (OECD, 2019). The trajectory of obesity begins early and becomes less malleable when children are older (Brown et al., 2019). Moreover, negative impacts of obesity in childhood track through into adulthood, thereby contributing to the greater risk of premature death and disability (WHO, 2020).

The global prevalence of childhood obesity is over 41 million in young children under the age 5 years (WHO, 